\title{
Preliminary Experimental Verification of Synthetic Aperture Flow Imaging Using a Dual
} Stage Beamformer Approach

\author{
Li, Ye; Jensen, Jørgen Arendt
}

Published in:

IFMBE Proceedings

Publication date:

2011

Link back to DTU Orbit

Citation (APA):

Li, Y., \& Jensen, J. A. (2011). Preliminary Experimental Verification of Synthetic Aperture Flow Imaging Using a Dual Stage Beamformer Approach. In K. Dremstrup, S. Rees, \& M. Ø. Jensen (Eds.), IFMBE Proceedings (Vol. 34, pp. 53-56). Springer.

- Users may download and print one copy of any publication from the public portal for the purpose of private study or research.

- You may not further distribute the material or use it for any profit-making activity or commercial gain

- You may freely distribute the URL identifying the publication in the public portal

If you believe that this document breaches copyright please contact us providing details, and we will remove access to the work immediately and investigate your claim. 


\title{
Preliminary Experimental Verification of Synthetic Aperture Flow Imaging Using a Dual Stage Beamformer Approach
}

\author{
Ye Li and Jørgen Arendt Jensen \\ Center for Fast Ultrasound Imaging, Dept. of Electrical Engineering, Technical University of Denmark, Lyngby, Denmark
}

\begin{abstract}
A dual stage beamformer method for synthetic aperture flow imaging has been developed. The motivation is to increase the frame rate and still maintain a beamforming quality sufficient for flow estimation that is possible to implement in a commercial scanner. With the new method high resolution images can be obtained continuously, which will highly increase the frame rate. The flow velocity is estimated by using a time-domain cross-correlation technique. The approach is investigated through experiments with the SARUS scanner (Synthetic Aperture Real-time Ultrasound System). A flow rig generates a parabolic laminar flow, and the SARUS scanner is used for acquiring the data from individual channels of the transducer. The experimental results showed that increasing the number of imaging lines used for the estimation form 4 to 24 reduces the standard deviation from $21 \%$ to $7.6 \%$. The parameter study showed that the number of crosscorrelation functions for averaging and length of the search range influence the performance.
\end{abstract}

Keywords - Synthetic aperture flow imaging, synthetic aperture sequential beamforming, SARUS.

\section{INTRODUCTION}

Synthetic aperture (SA) ultrasound imaging has many benefits compared to conventional imaging methods. Due to the complete data set, it is possible to have both dynamic transmit and receive focusing to improve contrast and resolution, and the data can be beamformed in any direction at any position. Therefore, SA flow imaging can achieve flow velocity mapping with a high frame rate and high resolution. However, a full SA method needs to acquire individual element data, which consumes huge amounts of memory, if the number of emissions is high. The high number of calculations is a challenge and makes real time implementation expensive. A synthetic aperture sequential beamforming (SASB) approach has been suggested without the need for storing RF-data and with a reduced system complexity [1]. Compared to a full SA setup only a single RF-line is beamformed and stored for each emission. That reduces the number of calculations and complexity significantly.

The approach was developed and tested through simulations [2]. The results indicated that it was possible to employ a dual stage beamformer approach for synthetic aperture flow imaging [2]. The standard deviation on the estimates was found to be less than $3 \%$ (compared to the peak velocity).

In this paper, a preliminary experiment was made to verify the method. A flow rig system with a gear pump generates a parabolic laminar flow profile inside the tube. A commercial linear array scans the tube at a fixed angle. The SARUS (Synthetic Aperture Real-time Ultrasound System) [3] scanner system amplifies and digitizes the receiving signals and stores the data in the local memory. The stored data sets are processed off-line to estimate the flow velocity profile.

\section{Method FOR SASB FLOW IMAGiNG}

This section describes how the dual stage beamformer approach and synthetic aperture flow imaging work.

\section{A. Dual stage beamformer approach}

At every emission, a focused beam is transmitted and received by a subaperture. The transmit elements are Hamming weighted to reduce edge effects. In the first stage the data is beamformed at the same fixed focus in both transmit and receive. The constructed $\mathrm{B}$-mode RF lines are then input to a second beamformer, which is fully dynamically focused. In the second stage, the emit focus points are considered as virtual sources, since a spherical wave emanates from this point in a limited angular region.

In receiving beamforming, the time of flight (TOF) is calculated to find the correct signal samples. The round trip TOF is calculated by tracing the path of the ultrasound wave, which is

$$
t_{\text {tof }}=\frac{\left|\vec{r}_{v e}-\vec{r}_{e}\right| \pm\left|\vec{r}_{i p}-\vec{r}_{v e}\right| \pm\left|\vec{r}_{v e}-\vec{r}_{i p}\right| \pm\left|\vec{r}_{r}-\vec{r}_{v e}\right|}{C},
$$

where $\vec{r}_{v e}, \vec{r}_{e}, \vec{r}_{i p}$, and $\vec{r}_{r}$ are the positions of the virtual element, the emitting element, the imaging point, and the receive element, respectively. The virtual sources are at the same position as the focal points. The wave starts from the transmit origin to the imaging point through the transmit focal point and propagates back to the receive element through the receive focal point. 
The beamforming method is illustrated in Fig. 1. The image from the second stage is constructed by selecting samples corresponding to the same position from the first stage B-mode lines. Therefore, each high resolution image point is obtained by summing the corresponding low resolution image point in the first stage.

The wave beam pattern in Fig. 1 is determined by the opening angle $\alpha$, which can be expressed as

$$
\alpha=2 \arctan \frac{1}{2 F^{\#}},
$$

where $F^{\#}$ is the ratio of the focal point depth and the size of the active sub-aperture. The choice of focal point depth and the sub-aperture length can influence the transmitted wave field and the point spread function (PSF).

Transducer

\section{IIIIIIIIIIIIIIIIIIIII}

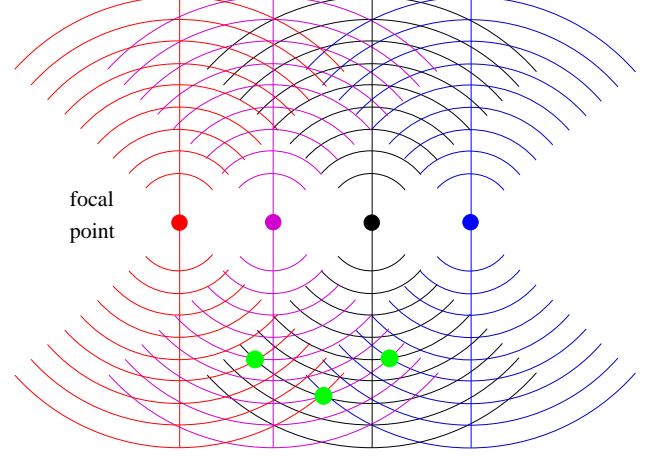

Fig. 1. Illustration of the second beamformer. Four image lines from the first stage are the inputs to the second stage here. They only carry information within the transmit pattern, which is defined by the opening angle. The high resolution points indicated as green dots are obtained by adding all low resolution points overlapping at that position.

\section{B. Velocity estimator}

At every emission the low resolution image is replaced by the latest one, thus, high resolution images can be obtained continuously. Due to the scatterer moving in a certain direction, the two consecutive signals are time shifted versions of each other, which can be expressed as [4]

$$
H^{n}(t)=H^{n-N}\left(t-2 \frac{v_{z}}{c} N T_{p r f}\right),
$$

where $t$ is the propagation time from pulse emission, $n$ is the global emission index, $N$ is the number of emissions in one sequence, $v_{z}$ is the velocity along the axial direction and $T_{\text {prf }}$ is the pulse repetition time.

The cross-correlation function is calculated between two high resolution images from the same emission sequence, which can be formulated as

$$
\begin{aligned}
R_{n-N, n}(\tau) & =\frac{1}{2 T} \int_{T} H^{(n-N)}(t) H^{(n)}(t+\tau) d t \\
& =\frac{1}{2 T} \int_{T} H^{(n-N)}(t) H^{(n-N)}\left(t-t_{s}+\tau\right) d t \\
& =R_{n-N, n-N}\left(\tau-t_{s}\right),
\end{aligned}
$$

where $t_{s}$ gives the times shift of the peak of the crosscorrelation function. The axial velocity can then be expressed as

$$
v_{z}=\frac{c t_{s}}{2 N T_{p r f}}
$$

The summation of several cross-correlation functions lowers the variance and improves the estimate. The velocity profile can be achieved by finding the cross-correlation function as a function of depths.

\section{EXPERIMENTAL SETUP}

The flow rig system is illustrated in Fig. 2. The fluid flows through the flow circulation system, which includes a gear pump drive, (Cole-Parmer, Model: 75211-15) that can vary the speed and a Danfoss MAGFLO Flowmeter (Type MAG 3000) for reference. The mean flow volume is measured by the flowmeter. An air-trap device removes air bubbles from the closed circuit. A $1.2 \mathrm{~m}$ long metal tube is used to develop the laminar flow, and the tube inside the water tank is made of rubber.

The concentrated Doppler fluid, (Dansk Fantom Service) consists of Glycerol, Orgasol, Triton X 100 (2\%), Nabenzoate, $\mathrm{Na}_{2}$ EDTA diluted 1:9 with demineralized water. Dextran is added to the solution to increase the viscosity as the flow phantom is intended to mimic the blood properties. The final kinematic viscosity of the flow phantom is 3.6 $\mathrm{mm}^{2} / \mathrm{s}$ which is within the acceptable range.

The measurement parameters can be found in Table 1. The angle between the ultrasound beam and the laminar flow is 70 degrees. The center of the tube is $28 \mathrm{~mm}$ from the surface transducer. The transmitting pulse and the data acquisition are performed by the SARUS scanner [7]. Arbitrary waveforms can be emitted and all channel data from the transducer are acquired simultaneously.

Each transmit sequence contains four emissions, which are active at different positions. The centers of the subapertures are several $\lambda$ away from each other. At each emissions, 64 elements are active and transmit a focused beam. The scattering signals are received by the same elements. The acquisition was made for 256 emissions using this transmit sequence, thus 252 high resolution lines can be 
obtained. The high resolution signal was convolved with a match filter to remove noise outside the pulse frequency range. Stationary echo cancelling was done by calculating the mean value of several high resolution signals and then subtracting this from the signals.

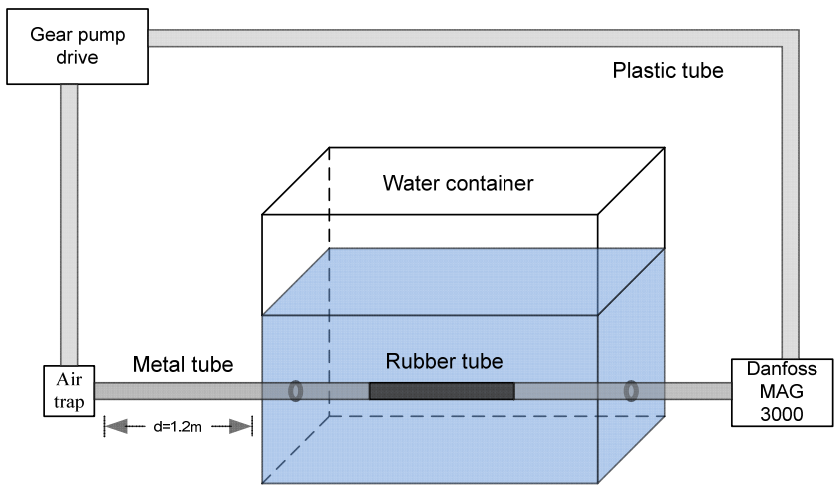

Fig. 2. Flow rig system. The flow is pumped out to the air trap to remove air bubbles, then moves through a $1.2 \mathrm{~m}$ long metal tube to develop a laminar flow. A flow meter measures the volume flow as a reference.

Table 1 Parameters for the measurement

\begin{tabular}{lll}
\hline \multicolumn{1}{c}{ Parameters } & Value & Unit \\
\hline Transducer center frequency & 7 & $\mathrm{MHz}$ \\
Sampling frequency & 70 & $\mathrm{MHz}$ \\
Transducer pitch & 0.208 & $\mathrm{~mm}$ \\
Transducer height & 4.5 & $\mathrm{~mm}$ \\
Pulse repetition frequency & 4 & $\mathrm{KHz}$ \\
Radius of rubber tube & 6.5 & $\mathrm{~mm}$ \\
Number of transmit element & 64 & \\
Number of receive element & 64 & \\
Focal depth (virtual source) & 10 & $\mathrm{~mm}$ \\
Volume flow speed & 20 & $\mathrm{~L} / \mathrm{h}$ \\
\hline
\end{tabular}

After applying the match filter and stationary echo cancelling, the signals were cross-correlated with the signals from the same sequence and added to the other cross correlation functions to obtain the velocity. Furthermore, the velocity profile is obtained as the cross-correlation was found as a function of depths.

The resulting image is shown in Fig. 3. There are four emissions with seven $\lambda$ spacing between each emission. 24 lines were used for each velocity estimate. The standard deviation varies between $1.8 \%$ and $20 \%$, with an average over the profile of $6.4 \%$ relative to the axial peak velocity of $0.034 \mathrm{~m} / \mathrm{s}$ and the average bias is $7.6 \%$ over the profile.

Fig. 4 shows the velocity profiles obtained from the same emission sequence, but with different number of averaging number. Averaging 48 cross-correlation functions yield better velocity estimates. The standard deviation and bias are $4.4 \%$ and $7.6 \%$ relative to the peak velocity.
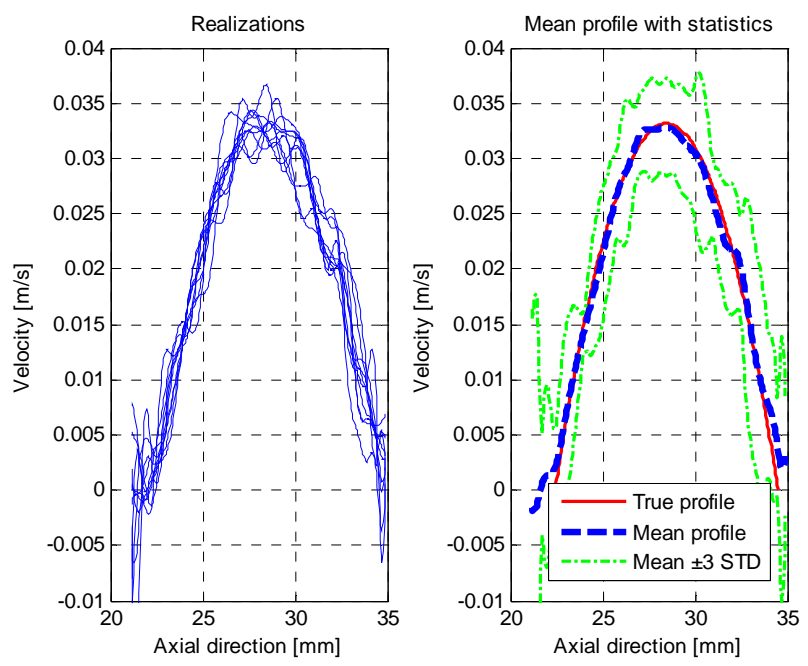

Fig. 3. Velocity profiles are obtained by four emissions with seven $\lambda$ spacing. 24 cross correlation functions are averaged to get one profile. The left graph shows 10 estimated profiles and the right shows the mean profile with \pm 3 standard deviations.
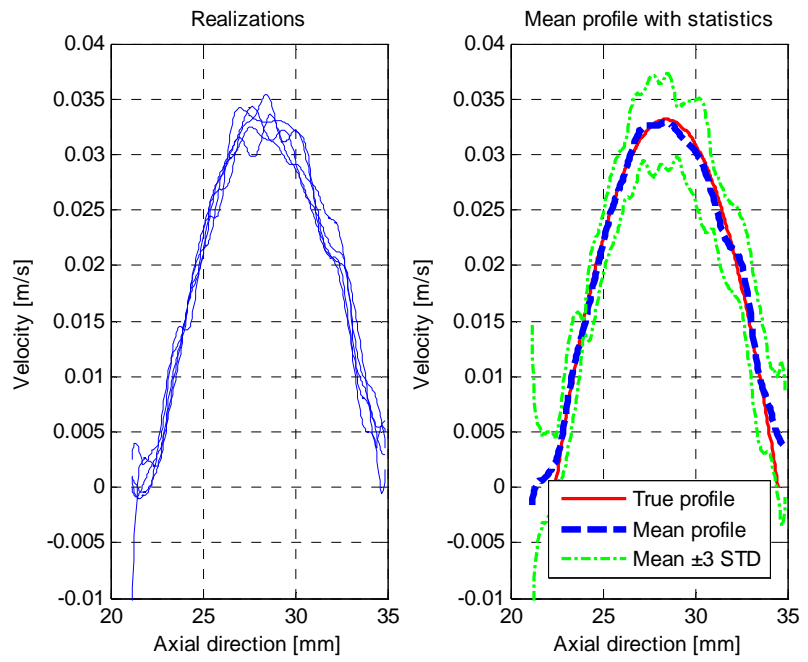

Fig. 4. Velocity profiles are obtained by four emissions with seven $\lambda$ spacing. 48 cross correlation functions are averaged to get one profile. The left graph shows 5 estimated profiles and the right shows the mean profile with \pm 3 standard deviations.

The results of the parameter study are shown in Fig. 5 and Fig. 6. It is obvious that both the averaging number and number of $\lambda$ in the searching range have an influence on the estimation performance. Using more cross correlation func- 
tions for one estimate gives better performance and a longer searching range gives more reliable results.

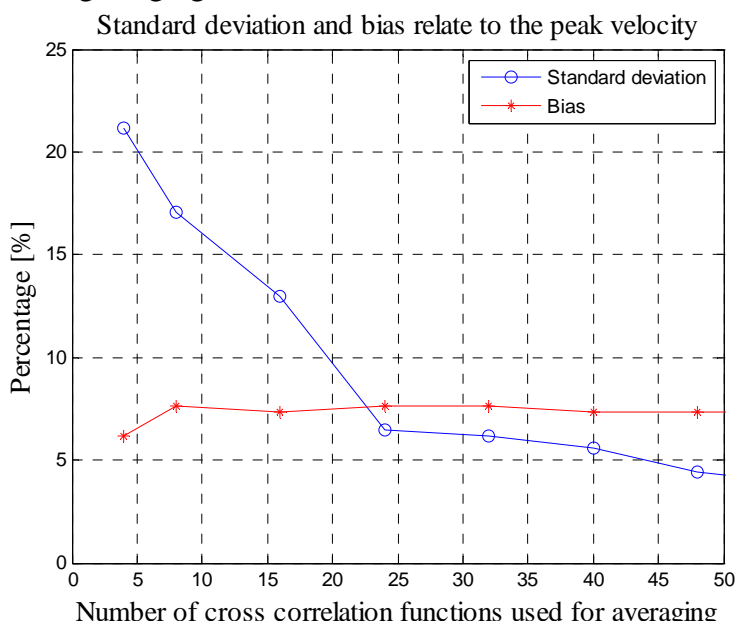

Fig. 5. The standard deviation and bias as a function of number of crosscorrelation functions averaged.

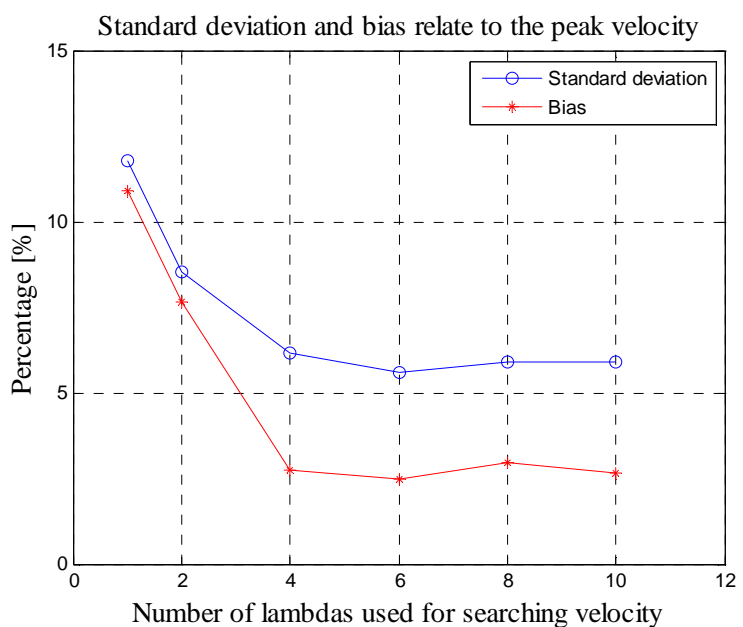

Fig. 6. The standard deviation and bias as a function of number of $\lambda$ in the search range.

\section{CONCLUSIONS}

The two stage beamformer approach is investigated experimentally for synthetic aperture flow imaging. Full synthetic aperture can acquire data continuously yielding high resolution images available for processing, and the data can be focused both in transmit and receive in any direction and position. However, this approach needs more allocated memory to store all channel data and demands more calculations. The new method has to store beamformed image lines from the first stage only instead of all the individual channel data. This reduces the number of calculations significantly and lowers the complexity of the system. Al- though the number of stored image lines is reduced, the beamforming quality is sufficient for flow imaging.

The preliminary experiment in this paper proved that it is possible to use the new method for synthetic aperture flow imaging, and that a fast flow imaging system can be built with the continuous data acquisition. Although a simple match filter was applied and mean subtraction used for stationary echo cancelling, the system is open for using other echo cancelling filters.

The SARUS scanner is not yet fully functioning and the data acquisition in this paper may be influenced by a low SNR (1.7 dB) due to low analogy amplification. Therefore, flow signals may at times have been affected by this, which makes the real signal difficult to detect.

The parameter study was done to examine how some key variables influence the estimation performance. The number of cross correlation functions used for averaging and the length of searching range influence the results, but in this preliminary experiment also the low SNR caused a decreased performance, when fewer emissions were averaged. Thus, the suggested method makes it possible to build a synthetic aperture flow imaging system with fewer calculations and a lower complexity of the system.

\section{AcKnowledgment}

This work is supported by grant 027-2008-3 from the Dasnish Advanced Technology Foundation.

\section{REFERENCES}

1. J. Kortbek and J. A. Jensen, "Synthetic aperture sequential beamforming,” in Proc. IEEE Ultrason. Symp., pp. 966-969, 2008.

2. Y. Li and J. A. Jensen, "Synthetic aperture flow imaging using a dual beamformer approach" in Proc. IEEE Ultrason. Symp., 2010.

3. J. A. Jensen, H. H. Lund, R. T. Nielson, B. G. Tomov, M. B. Stuart, S. I. Nikolov, M. Hansen and U. D. Larsen, "Performance of SARUS: A synthetic aperture real-time ultrasound system", in Proc. IEEE Ultrason. Symp., 2010.

4. S. I. Nikolov and J. A. Jensen, "In-vivo synthetic aperture flow imaging in medical ultrasound,” IEEE Trans. Ultrason., Ferroelec., Freq. Contr., vol. 39, pp. 366-380, 1992.

5. O. Bonnefous and P. Pesque, "Time domain formulation of pulse Doppler ultrasound and blood velocity estimation by cross correlation,” Ultrason. Imaging, vol. 8, pp 73-85, 1986.

6. P. M. Embree and W. D. O’Brien, "Volumetric blood flow via timedomain correlation: Experimental verification,” IEEE Trans. Ultrason., Ferrolelec., Freq. Contr., vol. 37, pp. 176-189, 1990a. 